\title{
Frequency of Nutritional Rickets in Breastfed Babies
}

\author{
Munazza Hayat, ${ }^{1}$ Muhammad Sajid, ${ }^{2}$ Naveed Akbar, ${ }^{3}$ Sana Sarwar, ${ }^{4}$ Maria Iftikhar, ${ }^{5}$ Zahid lqbal Sahi6 \\ 1,4,5Department of Pediatrics, Sir Ganga Ram Hospital, Lahore-Pakistan, 2Department of Pediatrics, Sheikh Zayed Medical College, Rahim Yar Khan-Pakistan, 3,6Department of Pediatrics \\ Fatima Jinnah Medical University, Lahore-Pakistan.
}

\section{ABSTRACT}

Background: Rickets is associated with biochemical abnormalities, bone deformities, impaired growth, developmental delays, and, late in the course of the disease, seizures. Several factors are thought to play a role in the rising incidence, including increased prevalence of prolonged breast-feeding, maternal vitamin $D$ deficiency, limited sunlight exposure and poor utilization of vitamin $D$ supplements. Objective: To determine the frequency of nutritional rickets in breastfed babies. Study Design: Descriptive Cross-Sectional study. Settings: Department of Pediatrics, Sir Ganga Ram Hospital, Lahore. Duration: From March 03, 2015 to September 02, 2015. Methodology: A total of 200 cases were included in the study. Nutritional rickets was assessed on X-ray wrist and venous blood sample was taken in an aseptic environment for measuring alkaline phosphatase by taking $1 \mathrm{ml}$ of blood and sent to the laboratory to be assessed on semi-automated micro-lab 300. Nutritional rickets was labeled as per operational definition. Results: Mean age of infants was 9.5+2.1 months. Mean gestational was $38.11 \pm 1.05$ weeks. In these 200 cases $134(67.0 \%)$ were male and $66(33.0 \%)$ female. While assessing the radiological signs of rickets it was noted that frequencies of radiological findings were as follows: metaphyseal fraying of distal radius/ulna $29(14.5 \%)$, metaphyseal cupping of distal radius/ulna $57(28.5 \%)$ and widening of distal end of metaphysis corresponding to thickened wrists or rachitic rosary $52(26.0 \%)$. Clinical findings were: frontal bossing $111(55.5 \%)$, fontanel widening $>2.5 \mathrm{~cm} 74(37.5 \%)$, wrist widening 108 $(54.0 \%)$ and rachitic rosary $13(6.5 \%)$. Positive biochemical findings were seen in 6 patients (3.0\%). Malnutrition was present in 98 infants (49.0\%). Conclusion: The study demonstrated Nutritional rickets was present in $36 \%$ of the breast-fed infants. Factors like low socioeconomic status and no formal education in this study were clearly associated with effected cases.

Keywords: Breastfed, Nutritional Rickets, Socioeconomic Status

Corresponding Author

Submitted for Publication: 01-01-2020

Dr. Muhammad Sajid, Assistant Professor, Department of Pediatrics, Sheikh Zayed Medical College, Rahim Yar Khan-Pakistan

Email: sajidaimc@gmail.com

Citation: Hayat M, Sajid M, Akbar N, Sarwar S, Iftikhar M, Sahi Zl. Frequency of Nutritional Rickets in Breastfed Babies. APMC 2020;14(2):115-8.

\section{INTRODUCTION}

Vitamin $\mathrm{D}$ deficiency in diet leads to nutritional Rickets. This is rare in developed countries but is prevalent in developing countries like Pakistan or counties at certain Latitude like northern latitudes. It is the fifth most common disease of developing region. ${ }^{1}$ This condition may result from prolonged breast feeding and is more common in children fed on vegetarian diet as a part of weaning. It is also seen in babies with dark skin pigmentation. There is also evidence of nutritional Rickets in population practicing certain cultural traditions like limited sun exposure of mother and breast-fed babies. ${ }^{2}$ There is little data present about its frequency in our population.

Vitamin D plays an important role in calcium hemostasis, normal bone development and maintenance. ${ }^{3}$ Nutritional rickets is a disease leading to bone pains and deformities, convulsions and delayed motor development .It is a disease of growing children. ${ }^{4}$ Bony deformities include bowing of legs, knock knees, Frontal bossing, widening at the end of long bones, rachitic rosary and pathological fractures in severe cases. ${ }^{1}$

According to one study conducted in Pakistan, Rickets was more common in age group of 2 to 12 months i.e. $79.8 \%$, and in those babies on breast feed (85.3\%). ${ }^{1}$ Another study found out that $23.9 \%$ children had nutritional rickets even in sun rich countries like Qatar. ${ }^{6}$ In one study most frequent presentation of nutritional rickets was hypokalemic or convulsions due to hypocalcemia $(34 \%){ }^{3}$

A study also showed that supplementation while breast feeding was necessary and those breastfed at one year of age who had not been supplemented with vitamin D had lower serum levels of $25-\mathrm{OHD}$ predisposing them to rickets. ${ }^{11}$

Rickets has different regimes of Vitamin D dose and treatment regimes. ${ }^{12-14}$

The purpose of this study is to determine the frequency of nutritional rickets in our population. There are a lot of studies that have described the consequences and complications of nutritional rickets but my study will focus on babies who are breastfed and are developing rickets. Literature search shows limited data on frequency of rickets in breast fed babies in Pakistan. Breast milk contains only $25 \mathrm{IU}$ per $1000 \mathrm{ml}$ of vitamin $D$, making it a very poor source in a population where exclusive breastfeeding is encouraged. This study will help us to see the frequency of nutritional rickets in our sun rich area thus making us to decide whether vitamin $D$ should be supplemented on a permanent basis to every breastfed baby that present in hospital, so in short if a high frequency is found then, this study will help to prevent permanent bone deformities and repeated hospital admissions by setting up guidelines for early detection and presentation of vitamin $\mathrm{D}$ deficiency. 


\section{OBJECTIVE}

To determine the frequency of nutritional rickets in breastfed babies.

\section{Operational Definitions}

Nutritional Rickets: A baby was labeled as nutritional rickets case if he/she has two of the three parameters from below:

Radiological Findings: Anyone of the following three signs assessed on X-ray wrist; Metaphyseal cupping (edge of metaphysis change from convex to concave), fraying (loss of sharp border of metaphysis) of the distal radius/ulna or widening of distal end of metaphysis.

Clinical Finding: Anyone of these clinical findings assessed by physician; Presence of frontal bossing, wrist widening, rachitic rosary (feels as beads of rosary at costochondral junction) or wide anterior fontanels more than $2.5 \mathrm{~cm}$, Harrison's groove or anterior bowing of tibia and femur.

Biochemical Finding: Raised alkaline phosphatase value more than $840 u / l$.

Breastfed Babies: Breastfed were defined as those babies who are exclusively breastfed in the first six month of life and were assessed by history from mother.

\section{METHODOLOGY}

Study Design: Descriptive cross-sectional study.

Settings: Department of Pediatrics, Sir Ganga Ram Hospital, Lahore Pakistan.

Duration: From March 03, 2015 to September 02, 2015.

Sample Technique: Non-probability consecutive sampling

Sample Size: A total of 200 babies was included.

Inclusion Criteria: Babies of age 6 months to 12 months on breast feed, both male and female. Babies presented for routine follow ups and vaccinations.

Exclusion Criteria: Babies with skeletal abnormalities as in osteogenesis imperfecta, achondroplasia, hyperparathyroidism, Down syndrome (on previous medical record) or babies with history of fits or those who were previously diagnosed to have fat malabsorption, liver disease or renal insufficiency (serum creatinine value more than $0.4 \mathrm{mg} / \mathrm{dl}$ ), babies who had received vitamin $D$ supplements in past six month or those with Previous hospital admissions and preterm babies were excluded from study.

Data Collection Procedure: An informed consent was taken from parents of babies before enrollment and filling out proforma especially designed for the study. Nutritional rickets was assessed on X-ray wrist and venous blood sample was taken in an aseptic environment for measuring alkaline phosphatase by taking $1 \mathrm{ml}$ of blood and sent to the laboratory to be assessed on semi-automated micro-lab 300. Nutritional rickets was labeled as per operational definition.

Data was entered and analyzed with the help of SPSS software 18. Descriptive statistics was used to calculate Mean and Standard Deviation for quantitative variables like age and serum alkaline phosphatase level. Frequency and percentage were calculated for qualitative variables like gender, nutritional status, radiological, and clinical findings. Data was stratified for age, gender, gestational age, socioeconomic status, nutritional status and health education to mothers to deal with effect modifiers. Post stratification Chi-square test was used. $P$ value $<0.05$ was considered significant.

\section{RESULTS}

The age group for this study was 6 to 12 months with mean $9.5+2.1$ months. Mean gestational age was $38.11 \pm 1.05$ weeks. In these 200 cases, 134 (67.0\%) were males and 66 (33.0\%) were females. While assessing the radiological signs of rickets it was noted that frequencies of radiological findings were as follows: metaphyseal fraying of distal radius/ulna $29(14.5 \%)$, metaphyseal cupping of distal radius/ulna $57(28.5 \%)$ and widening of distal end of metaphysis corresponding to thickened wrists or rachitic rosary 52 (26.0\%) Clinical findings were: frontal bossing $111(55.5 \%)$, fontanel widening $>2.5 \mathrm{~cm} 74(37.5 \%)$, wrist widening $108(54.0 \%)$ and rachitic rosary $13(6.5 \%)$ Positive biochemical findings were seen in 6 patients (3.0\%).Malnutrition was present in 98 infants (49.0\%). Nutritional rickets in breast-fed infants was present in 72 (36.0\%) cases Frequency of various clinical findings were calculated as shown in table 2. Frequency of radiological findings is shown in table 1.

Socioeconomic status showed that 107 cases $(53.7 \%)$ belonged to low income, 85 (42.5\%) belonged to middle income while only 8 cases $(4 \%)$ were having high income. Educational status of the mother revealed 85 mothers $(42.5 \%)$ had no formal education, 88 (44\%) had education until matric and 27 (13.5\%) had higher education.

Table 1: Distribution of cases by radiological findings

\begin{tabular}{|l|c|c|}
\hline Radiological findings & Number & Percentage \\
\hline $\begin{array}{l}\text { Metaphyseal fraying of distal } \\
\text { radius/ulna }\end{array}$ & 29 & 14.5 \\
\hline $\begin{array}{l}\text { Metaphyseal cupping of distal } \\
\text { radius/ulna }\end{array}$ & 57 & 28.5 \\
\hline $\begin{array}{l}\text { Widening of distal end of } \\
\text { metaphysis corresponding to } \\
\text { thickened wrists or rachitic rosary }\end{array}$ & 52 & 26.0 \\
\hline
\end{tabular}

Table 2: Distribution of cases by clinical findings

\begin{tabular}{|l|c|c|}
\hline Clinical findings & Number & Percentage \\
\hline Frontal bossing & 111 & 55.5 \\
\hline Fontanel widening $\mathbf{> 2 . 5} \mathbf{~ c m}$ & 75 & 37.5 \\
\hline Wrist widening & 108 & 54.0 \\
\hline Rachitic rosary & 13 & 6.5 \\
\hline
\end{tabular}

Total is not $100 \%$ as there were multiple responses

\section{DISCUSSION}

In this study all those babies were included who visited hospital for either vaccination or routine follow-up. No cases with complicated rickets were included in the study. All the infants 
were breast fed. It was seen in this study that $36 \%$ of the infants had nutritional rickets defined by the operational definition of the study. This study showed that there is a trend in male infants to be affected as seen in previous studies where the trend of male to female ratio was 2:1 which cannot be explained yet with no biological reason. ${ }^{3-6-7}$ In this study the ratio was 1:1.2 where male was $37 \%$ of the cases while female $33.3 \%$ of the cases. As there was a less trend of female patient in the study it needed to be further studied.

In this study it was also noticed that $1 / 3^{\text {rd }}$ of the cases had nutritional rickets making a large population of infants exposed to the complications of this disease. in a similar study conducted in Pakistan, it was noticed that $85.3 \%$ of breastfed babies had nutritional rickets. ${ }^{1}$ In a population that has not only higher skin pigmentation but has poor diet due to bad socioeconomic status, this study clearly showed that $1 / 3^{\text {rd }}$ of the infants that are breast fed are nutritionally deficient. ${ }^{1,2}$

With vitamin D supplementation the amount of vitamin $D$ in breast milk can be increased. ${ }^{3-5}$ But as seen in our population not only socioeconomic status is the hindrance but Health education of mothers is lacking in our society. In our study we noticed that mothers with no formal education had infants at a higher risk of rickets. Prolong breast feeding without any supplementation had been observed and concluded as the cause of nutritional rickets. ${ }^{3-5} \mathrm{~A}$ study in Saudi Arabia showed $55 \%$ of children with rickets were breast fed. ${ }^{15}$

The common finding observed was cupping of the ulna / radius occurring in $28.5 \%$ of the infants that were assessed followed by wrist widening and then fraying. In another study where wrist widening was most commonly occurring sign of rickets that is $26 \%$, the other physical findings like frontal bossing was $16 \%$ which is in contrast to our study having frontal bossing in $55.5 \%$ and wrist widening in $54 \%$ of cases. ${ }^{3}$

In this study the mean alkaline phosphatase was 476 with standard deviation of 324.3 , highlighting the fact that serum alkaline phosphatase levels should be monitored as it is least expensive and infant's serum alkaline phosphatase is derived from bones. ${ }^{3-8}$

Even though the city where this study was conducted has longer summers with adequate sun exposure in winters as well, but infants where still affected and had nutritional rickets. This led to observation that along with high pigmented skin that needs 3 to 6 times more sun exposure there was inadequate sun exposure in the infants. This may be due to the fact that houses are built in a way where there is less sun and ventilation along with insecurity to take infants out in the sun. Some of the cultural practices may also play role in inadequate exposure of babies to sun. ${ }^{6-9}$

The mother feed should be encouraged but mothers along with infants need to supplemented with vitamin D. ${ }^{3}$ Breast milk is a complete diet for an infant but in our country most of the mothers are deficient in vitamin $\mathrm{D}$.

In this study we were not able to do levels of 25-OHD which would have clearly established the serum levels in infant population along with mothers in this study were not assessed. As seen in other studies, hypovitaminosis still exist even in developed countries. ${ }^{9}$ Our country where we are still striving to achieve a state where food is available for everyone, vitamin $D$ fortification is an important step in that. As seen in this study mother's education and malnutrition along with socioeconomic status were clearly related to nutritional rickets so improving these factors will cause a direct effect on child nutritional status. Breast milk should be encouraged but mothers and infants need to be supplemented to prevent them from complications. One more aspect that needs to be seen is that exposure of an infant to sunlight should also be encouraged because exposure of a infant's head 20 minutes three times a week can produce sufficient vitamin D. ${ }^{3}$ Further studies need to be done where infants should be assessed for time of exposure to sunlight. Studies from other part of the world have shown that higher skin pigmentation requires more sun exposure than required in a Caucasian or white infant.

Further evaluation of mothers and infants will give us better presentation of nutritional rickets along with measurement of PTH and 25-OHD that haven't been done in this study. Supplementation should be started in a few days after birth and may continue for 12 months of age along with supplementation of the mothers. ${ }^{10}$

\section{CONCLUSION}

Study demonstrated Nutritional rickets in $36 \%$ of the breast-fed infants. One third of the infants that present for regular follow up are nutritionally deficient and to prevent the complications in such a large group of infants some guidelines should be made. To prevent vitamin $D$ deficiency in breast fed and dark-skinned infants due their cultural practices need to be supplemented according to American academy of pediatrics guidelines. The supplementation should start soon after birth and continue for the first 12 months of life. To prevent rickets infants should be supplemented with $400 \mathrm{IU} /$ day of vitamin D. If compliance is poor, infants should be assessed on each follow up for vaccination and advised accordingly. Adequate sun exposure should be advised for mothers and infants. The dosage and safety profile of vitamin $D$ should be studied to prevent complications of vit $d$ supplementation ad overdosage.

\section{LIMITATIONS}

One of the limitations of our study was that we did not follow up the cases.

\section{SUGGESTIONS / RECOMMENDATIONS}

we strongly recommend that alkaline phosphatase should be monitored in the follow up appointments of such babies.

\section{CONFLICT OF INTEREST / DISCLOSURE} None.

\section{ACKNOWLEDGEMENTS}

Authors have acknowledged their supervisor and seniors, for their guidelines and support. 


\section{REFERENCES}

1. Haider N, Nagi AG, Khan KM. Frequency of nutritional rickets in children admitted with severe pneumonia. J Pak Med Assoc. 2010;60(9):729-32.

2. Khan AS, Shah TH, Khan MA. Nutritional Rickets among Children in Peshawar, NWFP, Pakistan. Med Channel. 2009;15(2):135-9.

3. Al-Atawi MS, Al-Alwan I A, Al-Mutair A N, Tamim HM, Al-Jurayyan NA. Epidemiology of nutritional rickets in children. Saudi J Kidney Dis Transpl. 2009;20(2):260-5.

4. Shaw NJ, Mughal M. Vitamin D and child health part 1 (skeletal aspects). Arch Dis Child. 2013;98(5):363-7.

5. Wagner $\mathrm{CL}$, Greer F. Prevention of rickets and vitamin $\mathrm{D}$ deficiency in infants, children, and adolescents. Pediatrics. 2008;122(5):1142-52.

6. Molla A, Badawi MH. Risk factors for nutritional rickets among children in Kuwait. Pediatr Int. 2000;42(3):280-4.

7. Dawodu A, Agarwal M, Hossain M, Kochiyil J, Zayed R. Hypovitaminosis $D$ and vitamin $D$ deficiency in exclusively breastfeeding infants and their mothers in summer: A justification for vitamin $D$ supplementation of breast-feeding infants. J Pediatr. 2003;142(2):169-73.

8. Risteli L, Risteli J. Biochemical markers of bone metabolism. Ann Med. 1993;25(2):385-93.

9. Shah M, Salhab N, Patterson D, Seikaly MG. Nutritional rickets still afflicts children in North Texas. Tex Med. 2000;96(6):64-8.

10. Munns C, Zacharin MR, Rodda CP. Prevention and treatment of infant and childhood vitamin D deficiency in Australia and New Zealand: a consensus statement. Med J. 2006 4;185(5):268-72.

11. Darmawikarta D, Chen Y, Lebovic G, Birken CS, Parkin PC, Maguire JL. Total duration of breastfeeding, vitamin $d$ supplementation, and serum levels of 25 -Hydroxy vitamin D. Am J Public Health. 2016;106(4):714-9.

12. Spiller HA, Good TF, Spiller NE, Aleguas A. Vitamin D exposures reported to US poison centers 2000-2014: Temporal trends and outcomes. Hum Exp Toxicol 2016;35(5):457-61.

13. Dayre McNally J, lliriani K, Pojsupap S. Rapid Normalization of Vitamin D Levels: A Meta-Analysis. Paediatrics 2015;135(1):15266.

14. Priyambada L, Bhatia V, Singh N, Bhatia E. Serum 25 hydroxyvitamin $D$ profile after single large oral doses of cholecalciferol (vitamin D3) in medical staff in North India: a pilot study. J Postgrad Med. 2014;60(1):52-6.

15. Alshammari OMO, Almezani AMM,Alshlaqy AS, Alsiraa MNN, Alenazy GAG, AL Ghamdi SAS. The Prevalence of Rickets Disorder among Children in Saudi Arabia. EJHM. 2018;73(6):6943-7.

$\begin{array}{ll}\text { AUTHORSHIP CONTRIBUTION } \\ \text { Munazza Hayat } & \text { Main Author } \\ \text { Muhammad Sajid } & \text { Assistance for Data Collection } \\ \text { Naveed Akbar } & \text { Manuscript Writing } \\ \text { Sana Sarwar } & \text { References Citation } \\ \text { Maria Iftikhar } & \text { Literature Review } \\ \text { Zahid lqbal Sahi } & \text { Statistical Analysis }\end{array}$

